\title{
Gradient and Hessian Estimates for Dirichlet and Neumann Eigenfunctions*
}

\author{
Feng-Yu Wang \\ Center for Applied Mathematics, Tianjin University, Tianjin 300072, China \\ Department of Mathematics, Swansea University, Singleton Park, SA2 8PP, United Kingdom \\ wangfy@tju.edu.cn
}

April 14, 2019

\begin{abstract}
We establish integral formulas and sharp two-sided bounds for the Ricci curvature, mean curvature and second fundamental form on a Riemannian manifold with boundary. As applications, sharp gradient and Hessian estimates are derived for the Dirichlet and Neumann eigenfunctions.
\end{abstract}

AMS subject Classification: 58J32, 58J50.

Keywords: Ricci curvature, second fundamental form, mean curvature, eigenfunction, eigenvalue.

\section{Introduction}

Let $(M, \mathbf{g})$ be a $d$-dimensional complete connected Riemannian manifold with boundary $\partial M$, and let $N$ be the inward unit normal vector field of $\partial M$. We also denote $\mathbf{g}(u, v)=\langle u, v\rangle$ for two vector fields $u, v$. For $V \in C^{2}(M)$, let $L=\Delta+\nabla V$ and $\mu(\mathrm{d} x)=\mathrm{e}^{V(x)} \operatorname{vol}(\mathrm{d} x)$, where vol is the volume measure. Then $L$ is symmetric in $L^{2}(\mu)$ under the Neumann condition $\left(\left.N f\right|_{\partial M}=0\right)$ or the Dirichlet condition $\left(\left.f\right|_{\partial M}=0\right)$. We estimate the gradient and Hessian of the Dirichlet and Neumann eigenfunctions for $L$ by using the following quantities:

- Bakry-Emery curvature on $M: \mathcal{R} i c^{V}=\mathcal{R} i c-\operatorname{Hess}_{V}$, where $\mathcal{R} i c$ is the Ricci curvature.

- Second fundamental form of $\partial M: \mathbb{I}_{\partial}(u, v)=-\left\langle\nabla_{u} N, v\right\rangle, u, v \in T \partial M$.

${ }^{*}$ Supported in part by NNSFC (11771326, 11431014). 
- Weighted mean curvature of $\partial M: H_{V}=\operatorname{tr}\left(\mathbb{I}_{\partial}\right)-N V=-L \rho_{\partial}$ on $\partial M$, where $\rho_{\partial}$ is the Riemannian distance to the boundary $\partial M$. When $V=0, H_{0}$ is the usual mean curvature of $\partial M$.

Let $\mu_{\partial}$ be the area measure on $\partial M$ induced by $\mu$. For a nonnegative function $f$, we denote

$$
\mu(f)=\int_{M} f \mathrm{~d} \mu, \quad \mu_{\partial}(f)=\int_{\partial M} f \mathrm{~d} \mu_{\partial} .
$$

We call $(\lambda, \phi) \in(0, \infty) \times C^{2}(M)$ an eigenpair of $L$, if $L \phi=-\lambda \phi$ holds. Let $\operatorname{Eig}_{N}(L)$ be the set of eigenpairs $(\lambda, \phi)$ with $\mu\left(\phi^{2}\right)=1$ for the Neumann problem (i.e. $\left.N \phi\right|_{\partial M}=0$ ), and let $\operatorname{Eig}_{D}(L)$ be that for the Dirichlet eigenproblem (i.e. $\left.\phi\right|_{\partial M}=0$ ). We aim to estimate $\mu_{\partial}\left(\phi^{2}\right), \mu_{\partial}\left(|\nabla \phi|^{2}\right)$ and $\mu\left(\left\|\operatorname{Hess}_{\phi}\right\|_{H S}^{2}\right)$ for Dirichlet and Neumann eigenfunctions respectively. Before state our results, we first recall known boundary estimates derived in $[1,2]$.

According to [2], there exists a constant $C>0$ such that

$$
\mu_{\partial}\left(|\nabla \phi|^{2}\right) \leq C \lambda, \quad(\lambda, \phi) \in \operatorname{Eig}_{D}(\Delta)
$$

When $M$ does not contain any trapped geodesic, i.e. any geodesic starting from a point in $M$ will eventually go beyond $M$ (it is the case when $M$ is a domain in $\mathbb{R}^{d}$ ), then there exists a constant $c>0$ such that

$$
\mu_{\partial}\left(|\nabla \phi|^{2}\right) \geq c \lambda, \quad(\lambda, \phi) \in \operatorname{Eig}_{N}(\Delta)
$$

So, as a general result, the order of $\lambda$ in (1.1) is sharp. But, in general, (1.2) is not true, see [2] for counterexamples, which include semi-spheres and cylinders. This indicate that for boundary estimates of eigenfunctions, a smooth domain in $\mathbb{R}^{d}$ may be essentially different from a Riemannian manifold with boundary.

However, for Neumann eigenfunctions the estimate (1.1) does not hold. According to [1], when $M$ is a bounded smooth domain in $\mathbb{R}^{d}$ and $L=\Delta$, there exists a constant $C>0$ such that

$$
\mu_{\partial}\left(\phi^{2}\right) \leq C \lambda^{\frac{1}{3}}, \quad \mu_{\partial}\left(|\nabla \phi|^{2}\right) \leq C \lambda^{\frac{4}{3}}, \quad(\lambda, \phi) \in \operatorname{Eig}_{N}(\Delta)
$$

where the order of $\lambda$ in both estimates is sharp for the disc in $\mathbb{R}^{2}$.

In this paper, we aim to derive sharp Hessian estimate for $(\lambda, \phi) \in \operatorname{Eig}_{D}(L)$, and extend (1.3) to general compact Riemannian manifolds with boundary which, in turn, to imply sharp Hessian estimates for $(\lambda, \phi) \in \operatorname{Eig}_{N}(L)$.

We first consider the Hessian estimate for Dirichlet eigenfunctions. We will see that the following result is a straightforward consequence of (1.1) and the integral formula (2.2) proved in the next section.

Theorem 1.1 (Diriclet eigenfunctions). Let $M$ be a d-dimensional connected compact Riemannian manifold with boundary $\partial M$. Let $K_{1}, K_{2}, \delta_{1}, \delta_{2} \in \mathbb{R}$ be constants such that

$$
K_{1} \leq \mathcal{R} i c^{V} \leq K_{2}, \quad \delta_{1} \leq H_{V} \leq \delta_{2}
$$


Then

$$
K_{1} \lambda+\delta_{1} \mu_{\partial}\left(|\nabla \phi|^{2}\right) \leq \lambda^{2}-\mu\left(\left\|\operatorname{Hess}_{\phi}\right\|_{H S}^{2}\right) \leq K_{2} \lambda+\delta_{2} \mu_{\partial}\left(|\nabla \phi|^{2}\right), \quad(\lambda, \phi) \in \operatorname{Eig}_{D}(L)
$$

Consequently, there exists a constant $C>0$ such that

$$
\left|\mu\left(\left\|\operatorname{Hess}_{\phi}\right\|_{H S}^{2}\right)-\lambda^{2}\right| \leq C \lambda, \quad(\lambda, \phi) \in \operatorname{Eig}_{D}(L) .
$$

In particular, if $\mathcal{R} i c^{V}=0$ and $H_{V}=0$, then (1.5) holds for $C=0$.

Proof. Since $L \phi=-\lambda \phi,(1.4)$ follows from (2.2) in the next section. Next, by repeating the argument in [2], we may prove (1.1) for $\operatorname{Eig}_{D}(L)$ replacing $\operatorname{Eig}_{D}(\Delta)$. So, (1.5) follows from $(1.4)$.

By (1.4) and the sharpness of (1.1) as explained above, the order of $\lambda$ in estimate (1.5) is sharp as well.

The situation for the Neumann problem is more complicated. We address the main result below but leave the proof to Section 3 .

Theorem 1.2 (Neumann eigenfunctions). Let $M$ be a d-dimensional connected compact Riemannian manifold with boundary $\partial M$. Let $K_{1}, K_{2}, \kappa_{1}, \kappa_{2} \in \mathbb{R}$ be constants such that

$$
K_{1} \leq \mathcal{R} i c^{V} \leq K_{2}, \quad \kappa_{1} \leq \mathbb{I}_{\partial} \leq \kappa_{2}
$$

Then

$$
K_{1} \lambda+\kappa_{1} \mu_{\partial}\left(|\nabla \phi|^{2}\right) \leq \lambda^{2}-\mu\left(\left\|\operatorname{Hess}_{\phi}\right\|_{H S}^{2}\right) \leq K_{2} \lambda+\kappa_{2} \mu_{\partial}\left(|\nabla \phi|^{2}\right), \quad(\lambda, \phi) \in \operatorname{Eig}_{N}(L) .
$$

Moreover, there exists a constant $C>0$ such that

$$
\mu_{\partial}\left(\phi^{2}\right) \leq C \lambda^{\frac{1}{3}}, \quad \mu_{\partial}\left(|\nabla \phi|^{2}\right) \leq C \lambda^{\frac{4}{3}}, \quad(\lambda, \phi) \in \operatorname{Eig}_{N}(L)
$$

Consequently, there exists a constant $C>0$ such that

$$
\left|\mu\left(\left\|\operatorname{Hess}_{\phi}\right\|_{H S}^{2}\right)-\lambda^{2}\right| \leq C \lambda^{\frac{4}{3}}, \quad(\lambda, \phi) \in \operatorname{Eig}_{N}(L) .
$$

If in particular $\mathcal{R} i c^{V}=0$ and $\mathbb{I}_{\partial}=0$, (1.8) holds for $C=0$.

By (1.6) and the sharpness of (1.3) for the disc in $\mathbb{R}^{2}$ as explained in [1], the order of $\lambda$ in (1.8) is sharp as well.

In Section 2, we establish integral formulas and two-sided bounds for the above mentioned geometry quantities, which will be used in Section 3 to prove Theorem 1.2. 


\section{Integral characterizations of $\mathcal{R} i c^{V}, \mathbb{I}_{\partial}$ and $H_{V}$}

In this section, we assume that $M$ is a Riemannian manifold with boundary which is not necessarily compact nor connected. Let $C_{0}^{\infty}(M)$ be the set of smooth functions on $M$ with compact support. We consider the following two classes of reference functions for the Neumann and Dirichlet problems respectively:

$$
C_{N}^{\infty}(M):=\left\{f \in C_{0}^{\infty}(M):\left.N f\right|_{\partial M}=0\right\}, \quad C_{D}^{\infty}(M):=\left\{f \in C_{0}^{\infty}(M):\left.f\right|_{\partial M}=0\right\} .
$$

By Bochner-Weizenböck and integration by parts formulas, we have the following integral formulas for $\mathcal{R} i c^{V}, \mathbb{I}_{\partial}$ and $H_{V}$.

Theorem 2.1. Let $C_{N}^{\infty}(M)$ and $C_{D}^{\infty}(M)$ be in above. We have

$$
\begin{aligned}
& \int_{M}\left\{(L f)^{2}-\left\|\operatorname{Hess}_{f}\right\|_{H S}^{2}-\mathcal{R} i c^{V}(\nabla f, \nabla f)\right\} \mathrm{d} \mu=\int_{\partial M} \mathbb{I}_{\partial}(\nabla f, \nabla f) \mathrm{d} \mu_{\partial}, \quad f \in C_{N}^{\infty}(M) \\
& \int_{M}\left\{(L f)^{2}-\left\|\operatorname{Hess}_{f}\right\|_{H S}^{2}-\mathcal{R} i c^{V}(\nabla f, \nabla f)\right\} \mathrm{d} \mu=\int_{\partial M} H_{V}|\nabla f|^{2} \mathrm{~d} \mu_{\partial}, \quad f \in C_{D}^{\infty}(M) .
\end{aligned}
$$

Proof. By Bochner-Weizenböck formula,

$$
\frac{1}{2} L|\nabla f|^{2}=\left\|\operatorname{Hess}_{f}\right\|_{H S}^{2}+\langle\nabla L f, \nabla f\rangle+\mathcal{R} i c^{V}(\nabla f, \nabla f), \quad f \in C^{\infty}(M) .
$$

Next, the integration by parts formula gives

$$
\begin{gathered}
-\int_{M}\langle\nabla L f, \nabla f\rangle \mathrm{d} \mu=\int_{\partial M}(L f) N f \mathrm{~d} \mu_{\partial}+\int_{M}(L f)^{2} \mathrm{~d} \mu, \\
\frac{1}{2} \int_{M} L|\nabla f|^{2} \mathrm{~d} \mu=-\frac{1}{2} \int_{\partial M} N|\nabla f|^{2} \mathrm{~d} \mu_{\partial}=-\int_{\partial M} \operatorname{Hess}_{f}(\nabla f, N) \mathrm{d} \mu_{\partial} .
\end{gathered}
$$

Integrating (2.3) with respect to $\mathrm{d} \mu$ and using (2.4), (2.5), we arrive at

$$
\begin{aligned}
& \int_{M}\left\{(L f)^{2}-\left\|\operatorname{Hess}_{f}\right\|_{H S}^{2}-\mathcal{R} i c^{V}(\nabla f, \nabla f)\right\} \mathrm{d} \mu \\
& =\int_{\partial M}\left\{\operatorname{Hess}_{f}(N, \nabla f)-(N f) L f\right\} \mathrm{d} \mu_{\partial}, \quad f \in C_{0}^{\infty}(M) .
\end{aligned}
$$

With this formula we are able to prove (2.1) and (2.2) as follows.

Firstly, for $f \in C_{N}^{\infty}(M)$, we have $\left.N f\right|_{\partial M}=0$ and, by [3, the formula after (3.2)],

$$
\left.\operatorname{Hess}_{f}(N, \nabla f)\right|_{\partial M}=-\left.\left\langle\nabla_{\nabla f} N, \nabla f\right\rangle\right|_{\partial M}=\left.\mathbb{I}_{\partial}(\nabla f, \nabla f)\right|_{\partial M} .
$$

Then (2.1) follows from (2.6). 
Next, for $f \in C_{D}^{\infty}(M)$, we have $\left.f\right|_{\partial M}=0$. So, $\left.\nabla f\right|_{\partial M}=(N f) N$ and

$$
\left.\operatorname{Hess}_{f}(N, \nabla f)\right|_{\partial M}=\left.(N f) \operatorname{Hess}_{f}(N, N)\right|_{\partial M} .
$$

Let $\left\{v_{i}\right\}_{i=1}^{d-1}$ be orthonormal vector fields in a neighborhood of a point $x \in \partial M$, such that $\nabla v_{i}(x)=0$ and $\left\langle N, v_{i}\right\rangle(x)=0$. Then

$$
\Delta f(x)=\operatorname{Hess}_{f}(N, N)(x)+\sum_{i=1}^{d-1} \operatorname{Hess}_{f}\left(v_{i}, v_{i}\right)(x) .
$$

Combining this with $\left.\nabla f\right|_{\partial M}=\left.(N f) N\right|_{\partial M}$ and $\left\langle v_{i}, N\right\rangle(x)=0=\left\langle N, \nabla_{v_{i}} v_{i}\right\rangle(x)$, we arrive at

$$
\begin{aligned}
\Delta f(x)-\operatorname{Hess}_{f}(N, N)(x) & =\sum_{i=1}^{d-1} \operatorname{Hess}_{f}\left(v_{i}, v_{i}\right)(x)=\sum_{i=1}^{d-1} v_{i}\left\langle\nabla f, v_{i}\right\rangle(x) \\
& =\sum_{i=1}^{d-1} v_{i}\left\{(N f)\left\langle N, v_{i}\right\rangle\right\}(x)=\sum_{i=1}^{d-1}\left\{(N f)\left\langle\nabla_{v_{i}} N, v_{i}\right\rangle\right\}(x) \\
& =-\left\{(N f) \operatorname{tr}\left(\mathbb{I}_{\partial}\right)\right\}(x)=-\left(H_{0} N f\right)(x) .
\end{aligned}
$$

This, together with (2.7), yields

$$
\left.\operatorname{Hess}_{f}(N, \nabla f)\right|_{\partial M}=(N f)\left(\Delta f+H_{0} N f\right)=\left.\left\{H_{0}(N f)^{2}+(N f) \Delta f\right\}\right|_{\partial M} .
$$

Combining with $\left.\nabla f\right|_{\partial M}=\left.(N f) N\right|_{\partial M}$ leads to

$$
\begin{aligned}
& \left.\left\{\operatorname{Hess}_{f}(N, \nabla f)-(N f) L f\right\}\right|_{\partial M}=\left.\left\{H_{0}|\nabla f|^{2}-(N f)\langle\nabla V, \nabla f\rangle\right\}\right|_{\partial M} \\
& =\left.|\nabla f|^{2}\left(H_{0}-N V\right)\right|_{\partial M}=\left.H_{V}|\nabla f|^{2}\right|_{\partial M} .
\end{aligned}
$$

Substituting into (2.6), we prove (2.2).

We now characterize bounds of $\mathcal{R} i c^{V}, \mathbb{I}_{\partial}$ and $H_{V}$. For a symmetric 2 -tensor $Q$, we write $Q \geq 0$ (or $Q \leq 0$ ) if $Q(v, v) \leq 0$ (or $Q(v, v) \leq 0$ ) holds for all vectors $v$. For two symmetric 2-tensors $Q_{1}, Q_{2}$, we write $Q_{1} \geq Q_{2}$ (equivalently, $Q_{2} \leq Q_{1}$ ) if $Q_{1}-Q_{2} \geq 0$ (equivalently, $\left.Q_{2}-Q_{1} \leq 0\right)$.

Theorem 2.2. Let $Q$ and $Q_{\partial}$ be continuous symmetric 2-tensors on $M$ and $\partial M$ respectively, and let $q \in C(\partial M)$.

(1) $\mathcal{R} i c^{V} \geq Q$ and $\mathbb{I}_{\partial} \geq Q_{\partial}$ if and only if

$$
\begin{aligned}
& \int_{M}\left\{(L f)^{2}-\left\|\operatorname{Hess}_{f}\right\|_{H S}^{2}-Q(\nabla f, \nabla f)\right\} \mathrm{d} \mu \\
& \geq \int_{\partial M} Q_{\partial}(\nabla f, \nabla f) \mathrm{d} \mu_{\partial}, \quad f \in C_{N}^{\infty}(M) .
\end{aligned}
$$


(2) $\mathcal{R i c}^{V} \leq Q$ and $\mathbb{I}_{\partial} \leq Q_{\partial}$ if and only if

$$
\begin{aligned}
& \int_{M}\left\{(L f)^{2}-\left\|\operatorname{Hess}_{f}\right\|_{H S}^{2}-Q(\nabla f, \nabla f)\right\} \mathrm{d} \mu \\
& \leq \int_{\partial M} Q_{\partial}(\nabla f, \nabla f) \mathrm{d} \mu_{\partial}, \quad f \in C_{N}^{\infty}(M) .
\end{aligned}
$$

(3) $\mathcal{R}$ ic $V \geq Q$ and $H_{V} \geq q$ if and only if

$$
\begin{aligned}
& \int_{M}\left\{(L f)^{2}-\left\|\operatorname{Hess}_{f}\right\|_{H S}^{2}-Q(\nabla f, \nabla f)\right\} \mathrm{d} \mu \\
& \geq \int_{\partial M} q|\nabla f|^{2} \mathrm{~d} \mu_{\partial}, \quad f \in C_{D}^{\infty}(M) .
\end{aligned}
$$

(4) $\mathcal{R}$ ic $V$ $\leq Q$ and $H_{V} \leq q$ if and only if

$$
\begin{aligned}
& \int_{M}\left\{(L f)^{2}-\left\|\operatorname{Hess}_{f}\right\|_{H S}^{2}-Q(\nabla f, \nabla f)\right\} \mathrm{d} \mu \\
& \leq \int_{\partial M} q|\nabla f|^{2} \mathrm{~d} \mu_{\partial}, \quad f \in C_{D}^{\infty}(M) .
\end{aligned}
$$

Proof. According to Theorem 2.1, we only need to prove the sufficiency in all assertions.

According to (2.1) and (2.2), the inequalities (2.8) and (2.10) are equivalent to the following ones respectively:

$$
\begin{aligned}
& \int_{M}\left\{\mathcal{R} i c^{V}-Q\right\}(\nabla f, \nabla f) \mathrm{d} \mu+\int_{\partial M}\left\{\mathbb{I}_{\partial}-Q_{\partial}\right\}(\nabla f, \nabla f) \mathrm{d} \mu_{\partial} \geq 0, \quad f \in C_{N}^{\infty}(M), \\
& \int_{M}\left\{\mathcal{R} i c^{V}-Q\right\}(\nabla f, \nabla f) \mathrm{d} \mu+\int_{\partial M}\left\{\left(H_{V}-q\right)|\nabla f|^{2}\right\} \mathrm{d} \mu_{\partial} \geq 0, \quad f \in C_{D}^{\infty}(M) .
\end{aligned}
$$

By the following Lemma 3.2, the first implies $\mathcal{R} i c^{V} \geq Q$ and $\mathbb{I}_{\partial} \geq Q_{\partial}$, while the second yields $\mathcal{R} i c^{V} \geq Q$ and $H_{V} \geq q$. Thus, assertions (1) and (3) hold. Similarly, we can prove assertions (2) and (4).

Lemma 2.3. Let $Q, Q_{\partial}$ be continuous symmetric 2-tensors on $T M$ and $T \partial M$ respectively, and let $h \in C(\partial M)$.

(1) $Q \geq 0$ and $Q_{\partial} \geq 0$ if and only if

$$
\int_{M} Q(\nabla f, \nabla f) \mathrm{d} \mu+\int_{\partial M} Q_{\partial}(\nabla f, \nabla f) \mathrm{d} \mu_{\partial} \geq 0, \quad f \in C_{N}^{\infty}(M) .
$$

(2) $Q \geq 0$ and $h \geq 0$ if and only if

$$
\int_{M} Q(\nabla f, \nabla f) \mathrm{d} \mu+\int_{\partial M} h|\nabla f|^{2} \mathrm{~d} \mu_{\partial} \geq 0, \quad f \in C_{D}^{\infty}(M) .
$$


Proof. The necessity in these assertions are trivial. Below we prove the sufficiency.

(a) $Q \geq 0$. For $f \in C_{0}^{\infty}(M \backslash \partial M) \subset C_{N}^{\infty}(M) \cap C_{D}^{\infty}(M)$, we have $\left.\nabla f\right|_{\partial M}=0$ so that each of (2.12) and (2.13) implies

$$
\int_{M} Q(\nabla f, \nabla f) \mathrm{d} \mu \geq 0, \quad f \in C_{0}^{\infty}(M \backslash \partial M) .
$$

According to [4, Lemma 2.2] for $M \backslash \partial M$ replacing $M$, this implies $Q \geq 0$ in $M \backslash \partial M$. By the continuity of $Q$, it holds on $M$.

(b) $Q_{\partial} \geq 0$. Let $x_{0} \in \partial M$ and $X_{0} \in T_{x_{0}} \partial M$ with $\left|X_{0}\right|=1$, we aim to prove $Q_{\partial}\left(X_{0}, X_{0}\right) \geq$ 0 . To this end, we take the normal coordinates in a neighborhood $O\left(x_{0}\right)$ of $x_{0}$ such that

(1) $x_{0}=0 \in \mathbb{R}^{d}, X_{0}=\left.\partial_{1}\right|_{x=0}$;

(2) For some constant $r_{0}>0$,

$$
O\left(x_{0}\right)=\left\{\left(x^{1}, \cdots, x^{d}\right) \in \mathbb{R}^{d}: 0 \leq x^{d}, \sum_{i=1}^{d}\left|x^{i}\right|^{2}<r_{0}\right\}
$$

(3) $(\partial M) \cap O\left(x_{0}\right)=\left\{x=\left(x^{1}, \cdots, x^{d}\right) \in O\left(x_{0}\right): x^{d}=0\right\}$, on which $N=\partial_{d}$.

Under this local coordinate system, let $\hat{x}=\left(x^{1}, \cdots, x^{d-1}, 0\right)$ for $x=\left(x^{1}, \cdots, x^{d}\right)$. Then there exist symmetric matrix-valued continuous functions $\left(q^{i j}\right)_{1 \leq i, j \leq d}$ and $\left(q_{\partial}^{i j}\right)_{1 \leq i, j \leq d-1}$ such that

$$
\begin{aligned}
& Q(\nabla f, \nabla f) \mathrm{d} \mu=\sum_{i, j=1}^{d}\left\{q^{i j}\left(\partial_{i} f\right)\left(\partial_{j} f\right)\right\}(x) \mathrm{d} x \text { on } O\left(x_{0}\right), \\
& Q_{\partial}(\nabla f, \nabla f) \mathrm{d} \mu_{\partial}=\sum_{i, j=1}^{d-1}\left\{q_{\partial}^{i j}\left(\partial_{i} f\right)\left(\partial_{j} f\right)\right\}(\hat{x}) \mathrm{d} \hat{x} \text { on }(\partial M) \cap O\left(x_{0}\right) .
\end{aligned}
$$

Now, for any $n \geq 1$ and $x \in \mathbb{R}^{d}$, let

$$
\left(\phi_{n}(x)\right)^{i}= \begin{cases}n^{2} x^{1}, & i=1, \\ n x^{i}, & 2 \leq i \leq d .\end{cases}
$$

Let $f \in C_{0}^{\infty}\left(O\left(x_{0}\right)\right)$ with $\left.N f\right|_{\partial M}=0$, i.e. $\left.\partial_{d} f\right|_{x^{d}=0}=0$. Then

$$
f_{n}:=f \circ \phi_{n} \in C_{0}^{\infty}\left(O\left(x_{0}\right)\right),\left.\quad \partial_{d} f_{n}\right|_{x^{d}=0}=0, \quad n \geq 1 .
$$

So, by (2.12) and (2.14) we obtain

$$
\begin{aligned}
0 & \leq \int_{\mathbb{R}^{d}} \sum_{i, j=1}^{d}\left\{q^{i j}\left(\partial_{i} f_{n}\right)\left(\partial_{j} f_{n}\right)\right\}(x) \mathrm{d} x+\int_{\mathbb{R}^{d-1}} \sum_{i, j=1}^{d-1}\left\{q_{\partial}^{i j}\left(\partial_{i} f_{n}\right)\left(\partial_{j} f_{n}\right)\right\}(\hat{x}) \mathrm{d} \hat{x} \\
& =\int_{\mathbb{R}^{d}}\left\{n^{1-d} \sum_{i, j=2}^{d}\left(q^{i j} \circ \phi_{n}^{-1}\right)\left(\partial_{i} f\right)\left(\partial_{j} f\right)+2 n^{2-d} \sum_{j=2}^{d}\left(q^{1 j} \circ \phi_{n}^{-1}\right)\left(\partial_{1} f\right)\left(\partial_{j} f\right)\right.
\end{aligned}
$$




$$
\begin{gathered}
\left.+n^{3-d}\left(q^{11} \circ \phi_{n}^{-1}\right)\left(\partial_{1} f\right)^{2}\right\}(x) \mathrm{d} x \\
+\int_{\mathbb{R}^{d-1}}\left\{n^{2-d} \sum_{i, j=2}^{d-1}\left(q_{\partial}^{i j} \circ \phi_{n}^{-1}\right)\left(\partial_{i} f\right)\left(\partial_{j} f\right)+2 n^{3-d} \sum_{j=2}^{d-1}\left(q_{\partial}^{1 j} \circ \phi_{n}^{-1}\right)\left(\partial_{1} f\right)\left(\partial_{j} f\right)\right. \\
\left.+n^{4-d}\left(q_{\partial}^{11} \circ \phi_{n}^{-1}\right)\left(\partial_{1} f\right)^{2}\right\}(\hat{x}) \mathrm{d} \hat{x} .
\end{gathered}
$$

Multiplying by $n^{d-4}$ and letting $n \rightarrow \infty$, we arrive at

$$
0 \leq q_{\partial}^{11}(0) \int_{\mathbb{R}^{d-1}}\left(\partial_{1} f\right)^{2}(\hat{x}) \mathrm{d} \hat{x}, \quad f \in(\partial M) \cap C_{0}^{\infty}\left(O\left(x_{0}\right)\right) .
$$

Combining this with the second equality in (2.14) and noting that $X_{0}=\left.\partial_{1}\right|_{x_{0}}$, we obtain $Q_{\partial}\left(X_{0}, X_{0}\right) \geq 0$.

(c) $h \geq 0$. Let $g \in C_{0}^{\infty}(\partial M)$ with compact support $D \subset \partial M$. There exist a neighborhood $\mathscr{O}$ in $M$ of $D$, and a constant $r_{0}>0$, such that $\rho_{\partial} \in C_{b}^{\infty}(\mathscr{O})$ and the Fermi coordinates

$$
\mathscr{O} \ni x=(\theta, r) \in \partial M \times\left[0, r_{0}\right)
$$

exists, where $x=(\theta, r)$ means $x=\exp _{\theta}[r N]$. Let $\gamma \in C_{0}^{\infty}([0, \infty))$ such that $\left.\gamma\right|_{\left[0, r_{0} / 2\right]}=$ $1,\left.\gamma\right|_{\left[r_{0}, \infty\right)}=0$. For any $n \geq 1$, define

$$
f_{n}(x):= \begin{cases}g(\theta) r \gamma(n r), & \text { if } x=(\theta, r) \in \mathscr{O} \\ 0, & \text { otherwise }\end{cases}
$$

Then $f_{n} \in C_{D}^{\infty}(M)$ and

$$
\left.\left|\nabla f_{n}\right|^{2}\right|_{\partial M}=\left.g^{2}\right|_{\partial M}, \quad\left|\nabla f_{n}\right| \leq c\left\{\left\|\nabla^{\partial M} g\right\|_{\infty}+\|g\|_{\infty}\left(1+r_{0}\left\|\gamma^{\prime}\right\|_{\infty}\right)\right\} 1_{\left\{\rho_{\partial} \leq r_{0} / n\right\}},
$$

where $\nabla^{\partial M}$ is the gradient on $\partial M$. So, applying (2.13) for $f_{n}$ replacing $f$ we may find out a constant $C>0$ such that for any $n \geq 1$,

$$
\begin{aligned}
& \int_{\partial M}\left(h g^{2}\right) \mathrm{d} \mu_{\partial} \geq-\int_{M} Q\left(\nabla f_{n}, \nabla f_{n}\right) \mathrm{d} \mu \\
& \geq-C \int_{\left\{\rho_{\partial} \leq r_{0} / n\right\} \cap \mathscr{O}}\left\{\left\|\nabla^{\partial M} g\right\|_{\infty}^{2}+\|g\|_{\infty}^{2}\left(1+r_{0}\left\|\gamma^{\prime}\right\|_{\infty}\right)^{2}\right\} \mathrm{d} \mu .
\end{aligned}
$$

By letting $n \rightarrow \infty$ we arrive at

$$
\int_{\partial M}\left(h g^{2}\right) \mathrm{d} \mu_{\partial} \geq 0, \quad g \in C_{0}^{\infty}(\partial M),
$$

which implies $h \geq 0$ as $g \in C_{0}^{\infty}(\partial M)$ is arbitrary. 


\section{Proof of Theorem 1.2}

To prove Theorem 1.2, we present some lemmas.

Lemma 3.1. There exists a constant $C>0$ such that

$$
\int_{\partial M}|\nabla \phi|^{2} \mathrm{~d} \mu_{\partial} \leq C\left(\lambda+\lambda \int_{\partial M} \phi^{2} \mathrm{~d} \mu_{\partial}\right), \quad(\lambda, \phi) \in \operatorname{Eig}_{N}(L) .
$$

Proof. Let $r_{0}>0$ such that $\rho_{\partial}$ is smooth on $\partial_{r_{0}} M:=\left\{\rho_{\partial} \leq r_{0}\right\}$ and the Fermi coordinate system $x=(\theta, r) \in \partial M \times\left[0, r_{0}\right]$ exists on $M_{0, r_{0}}:=\left\{\rho_{\partial} \leq r_{0}\right\}$. Under this coordinate system we have

$$
L=\alpha\left\{\Delta_{\partial M}+\nabla^{\partial M} V\right\}+\partial_{r}^{2}+Z
$$

where $\alpha \in C^{\infty}\left(\partial M \times\left[0, r_{0}\right]\right)$ is strictly positive with $\alpha(\cdot, 0)=1, \Delta_{\partial M}$ and $\nabla^{\partial M}$ are the Laplacian and gradient on the $(d-1)$-dimensional Riemannian manifold $\partial M$ respectively, and $Z$ is a $C^{1}$ (hence, bounded) vector field on $M$. Using the integration by parts formula on $\partial M,(3.1)$, and $L \phi=-\lambda \phi$, we obtain

$$
\begin{aligned}
& \int_{\partial M}|\nabla \phi|^{2} \mathrm{~d} \mu_{\partial}=-\int_{\partial M} \phi\left\{\Delta_{\partial}+\nabla^{\partial M} V\right\} \phi \mathrm{d} \mu_{\partial} \\
& =\int_{\partial M}\left\{\phi \operatorname{Hess}_{\phi}(N, N)+\phi Z \phi-\phi L \phi\right\} \mathrm{d} \mu_{\partial} \\
& \leq\left(\lambda+\|Z\|_{\infty}^{2}\right) \int_{\partial M} \phi^{2} \mathrm{~d} \mu_{\partial}+\frac{1}{4} \int_{\partial M}|\nabla \phi|^{2} \mathrm{~d} \mu_{\partial}+\int_{\partial M} \phi \operatorname{Hess}_{\phi}(N, N) \mathrm{d} \mu_{\partial} .
\end{aligned}
$$

Since $\lambda \geq \lambda_{1}^{N}>0$, this implies

$$
\int_{\partial M}|\nabla \phi|^{2} \mathrm{~d} \mu_{\partial} \leq c_{1} \lambda \int_{\partial M} \phi^{2} \mathrm{~d} \mu_{\partial}+\frac{4}{3} \int_{\partial M} \phi \operatorname{Hess}_{\phi}(N, N) \mathrm{d} \mu_{\partial}, \quad(\lambda, \phi) \in \operatorname{Eig}_{N}(L)
$$

for some constant $c_{1}>0$. To estimate $\int_{\partial M} \phi \operatorname{Hess}_{\phi}(N, N) \mathrm{d} \mu_{\partial}$, we take $\gamma \in C_{0}^{\infty}([0, \infty))$ such that $\left.\gamma\right|_{\left[0, r_{0} / 2\right]}=1,\left.\gamma\right|_{\left[2 r_{0} / 3, \infty\right)}=0$. By $L \phi=-\lambda \phi,\left.N \phi\right|_{\partial M}=0$ and using integration by parts, we have

$$
\begin{aligned}
& \int_{\partial M} \phi \operatorname{Hess}_{\phi}(N, N) \mathrm{d} \mu_{\partial}=\int_{\partial M} \phi N\left\langle\gamma\left(\rho_{\partial}\right) \nabla \rho_{\partial}, \nabla \phi\right\rangle \mathrm{d} \mu_{\partial} \\
& =\int_{M}\left\{-\phi L\left\langle\gamma\left(\rho_{\partial}\right) \nabla \rho_{\partial}, \nabla \phi\right\rangle+(L \phi)\left\langle\gamma\left(\rho_{\partial}\right) \nabla \rho_{\partial}, \nabla \phi\right\rangle\right\} \mathrm{d} \mu \\
& =\int_{M} \phi\left[\gamma\left(\rho_{\partial}\right) \nabla \rho_{\partial}, L\right] \phi \mathrm{d} \mu,
\end{aligned}
$$

where $\left[\gamma\left(\rho_{\partial}\right) \nabla \rho_{\partial}, L\right]:=\left(\gamma\left(\rho_{\partial}\right) \nabla \rho_{\partial}\right) L-L\left(\gamma\left(\rho_{\partial}\right) \nabla \rho_{\partial}\right)$ is a continuous second order differential operator on the compact set $\left\{\rho_{\partial} \leq r_{0}\right\}$. Combining this with $\int_{M} \phi^{2} \mathrm{~d} \mu=1$, we derive

$$
\left|\int_{\partial M} \phi \operatorname{Hess}_{\phi}(N, N) \mathrm{d} \mu_{\partial}\right| \leq c_{2}\left(\int_{M}\left(\phi^{2}+|\nabla \phi|^{2}+\left\|\operatorname{Hess}_{\phi}\right\|_{H S}^{2}\right) \mathrm{d} \mu\right)^{\frac{1}{2}}
$$


for some constant $c_{2}>0$. Combining with (3.2), $\mu\left(\phi^{2}\right)=1$ and $\mu\left(|\nabla \phi|^{2}\right)=\lambda$, we arrive at

$$
\int_{\partial M}|\nabla \phi|^{2} \mathrm{~d} \mu_{\partial} \leq c_{1} \lambda \int_{\partial M} \phi^{2} \mathrm{~d} \mu_{\partial}+\frac{4 c_{2}}{3}\left(1+\lambda+\int_{\partial M}\left\|\operatorname{Hess}_{\phi}\right\|_{H S}^{2} \mathrm{~d} \mu\right)^{\frac{1}{2}} .
$$

But by (1.6) we have

$$
\int_{\partial M}\left\|\operatorname{Hess}_{\phi}\right\|_{H S}^{2} \mathrm{~d} \mu \leq \lambda^{2}+c_{3} \lambda+\int_{\partial M}|\nabla \phi|^{2} \mathrm{~d} \mu_{\partial}
$$

for some constant $c_{3}>0,(3.5)$ implies the desired estimate for some constant $C>0$.

Lemma 3.2. There exists a constant $C>0$ such that

$$
\int_{\partial M} \phi^{2} \mathrm{~d} \mu_{\partial} \leq C \lambda^{\frac{1}{3}}, \quad(\lambda, \phi) \in \operatorname{Eig}_{N}(L)
$$

We first prove a priori estimate then make improvement. To this end, we introduce some notation.

For any $r \geq 0$, let $\partial_{r} M=\left\{\rho_{\partial}=r\right\}$ and $\mu_{\partial}^{r}$ be the area measure on it induced by $\mu$. For $0<r_{1}<r_{2}$, let

$$
M_{r_{1}, r_{2}}=\left\{r_{1} \leq \rho_{\partial} \leq r_{2}\right\}, \quad \mu_{\partial}^{r_{1}, r_{2}}=1_{\partial_{r_{1}} M} \mu_{\partial}^{r_{1}}+1_{\partial_{r_{2}} M} \mu_{\partial}^{r_{2}}
$$

Obviously, $\partial_{0} M=\partial M, \mu_{\partial}^{0}=\mu_{\partial}$.

Let $\delta>0$ such that $\rho_{\partial} \in C_{b}^{\infty}\left(M_{0, \delta}\right)$, and the Fermi coordinate system $(\theta, r) \in \partial M \times[0, \delta]$ gives a diffeomorphism between $M_{0, \delta}$ and $\partial M \times[0, \delta]$. Under this coordinate system we have

$$
\mu(\mathrm{d} \theta, \mathrm{d} r)=\psi(\theta, r) \mu_{\partial}(\mathrm{d} \theta) \mathrm{d} r
$$

for some strictly positive function $\psi \in C_{b}^{\infty}(\partial M \times[0, \delta])$. In particular, there exists a constant $c_{0}>0$ such that

$$
\int_{\partial M \times[0, \delta]}|f(\theta, r)|^{2} \mu_{\partial}(\mathrm{d} \theta) \mathrm{d} r \leq c_{0} \int_{M} f^{2} \mathrm{~d} \mu, \quad f \in \mathscr{B}(M) .
$$

Lemma 3.3. There exists a constant $c>0$ such that

$$
\int_{\partial_{r} M} \phi^{2} \mathrm{~d} \mu_{\partial}^{r} \leq c \sqrt{\lambda} \int_{M} \phi^{2} \mathrm{~d} \mu, \quad(\lambda, \phi) \in \operatorname{Eig}(L):=\operatorname{Eig}_{N}(L) \cup \operatorname{Eig}_{D}(L), r \in[0, \delta] .
$$

Proof. By the symmetry, we only prove the inequality for $r \in[0, \delta / 2]$. For $r \in[0, \delta / 2]$, define

$$
\gamma(s)=\frac{\delta-r}{\pi} \sin \left(\frac{(s-r) \pi}{\delta-r}\right), \quad s \in[r, \delta] .
$$

Then $\left|\nabla \gamma\left(\rho_{\partial}\right)\right| \leq 1$ and

$$
\sup _{M_{r, \delta}}\left|L \gamma\left(\rho_{\partial}\right)\right| \leq \sup _{M_{r, \delta}}\left|L \rho_{\partial}\right|+\frac{\pi}{\delta-r} \leq \sup _{M_{r, \delta}}\left|L \rho_{\partial}\right|+\frac{2 \pi}{\delta}=: c_{0}<\infty .
$$


Let $N$ be the inward normal unit vector field of $\partial M_{r, \delta}$. Then

$$
\left.N \gamma\left(\rho_{\partial}\right)\right|_{\partial M_{r, \delta}}=\gamma^{\prime}(r) 1_{\left\{\rho_{\partial}=r\right\}}-\gamma^{\prime}(\delta) 1_{\left\{\rho_{\partial}=r\right\}}=1_{\partial M_{r, \delta}} .
$$

So, by integration by parts, there exists a constant $c>0$ such that for any $(\phi, \lambda) \in \operatorname{Eig}(L)$,

$$
\begin{aligned}
& \int_{\partial_{r} M} \phi^{2} \mathrm{~d} \mu_{\partial}^{r}+\int_{\partial_{\delta} M} \phi^{2} \mathrm{~d} \mu_{\partial}^{\delta}=\int_{\partial M_{r, \delta}} \phi^{2} N \gamma\left(\rho_{\partial}\right) \mathrm{d} \mu_{\partial}^{r, \delta} \\
& =-\int_{M_{r, \delta}}\left\{\phi^{2} L \gamma\left(\rho_{\partial}\right)+\left\langle\nabla \gamma(\rho), \nabla \phi^{2}\right\rangle\right\} \mathrm{d} \mu \\
& \leq \int_{M}\left(c \phi^{2}+|\phi| \cdot|\nabla \phi|\right) \mathrm{d} \mu \leq c+\sqrt{\mu\left(\phi^{2}\right) \mu\left(|\nabla \phi|^{2}\right)}=c+\sqrt{\lambda}
\end{aligned}
$$

Combining (1.6) with Lemmas 3.1 and 3.3, we conclude that

$$
\left|\mu\left(\left\|\operatorname{Hess}_{\phi}\right\|_{H S}^{2}\right)-\lambda^{2}\right| \leq c \lambda^{\frac{3}{2}}, \quad(\lambda, \phi) \in \operatorname{Eig}_{N}(L) .
$$

Lemma 3.4. For any $(\phi, \lambda) \in \operatorname{Eig}_{N}(L)$, under the Fermi coordinates $(\theta, \lambda) \in \partial M \times[0, \delta]$ let

$$
h(r)=\frac{1}{\lambda} \int_{\partial M} \phi^{2}(\theta, r) \mu_{\partial}(\mathrm{d} \theta), \quad r \in[0, \delta] .
$$

Then there exists a constant $C>0$ depending only on $M_{0, \delta}$ and $L$ such that for $r \in[0, \delta]$ with $h^{\prime}(r) \geq 0$,

$$
h^{\prime \prime}(r) \geq \frac{\left|h^{\prime}(r)\right|^{2}}{h(r)}-C .
$$

Proof. (1) Obviously, we have $h^{\prime}(r)=\frac{2}{\lambda} \int_{\partial M} \phi \partial_{r} \phi \mathrm{d} \mu_{\partial}$ and

$$
h^{\prime \prime}(r)=\frac{2}{\lambda} \int_{\partial M}\left\{\left(\partial_{r} \phi\right)^{2}+\phi \partial_{r}^{2} \phi\right\}(\cdot, r) \mathrm{d} \mu_{\partial}, \quad r \in[0, \delta] .
$$

Let $N_{0, r}$ be the inward unit normal vector field of $\partial M_{0, r}=\left\{\rho_{\partial}=0\right\} \cup\left\{\rho_{\partial}=r\right\}$. Noting that $\left.\partial_{r} \phi\right|_{r=0}=\left.N \phi\right|_{\partial M}=0$, and $L \phi=-\lambda \phi$ implies

$$
\phi \psi^{-1}\left[\partial_{r}, L\right] \phi=(L \phi) \psi^{-1} \partial_{r} \phi-\phi \psi^{-1} L\left(\partial_{r} \phi\right),
$$

where $\left[\partial_{r}, L\right]:=\partial_{r} L-L \partial_{r}$ is a continuous second order differential operator on the compact set $\left\{\rho_{\partial} \leq \delta\right\}$, by (3.6) and the integration by parts formula, we have

$$
\begin{aligned}
& \frac{2}{\lambda} \int_{\partial M}\left(\left\{\phi \partial_{r}^{2} \phi\right\}(\cdot, r)+\left\{\phi N^{2} \phi\right\}(\cdot, 0)\right) \mathrm{d} \mu_{\partial}=\frac{2}{\lambda} \int_{\partial M_{0, r}}\left\{\phi \psi^{-1} N_{0, r} \partial_{r} \phi\right\} \mathrm{d} \mu_{\partial}^{0, r} \\
& =\frac{2}{\lambda} \int_{M_{0, r}}\left(\phi \psi^{-1} L\left(\partial_{r} \phi\right)-\left\{L\left(\phi \psi^{-1}\right)\right\} \partial_{r} \phi\right) \mathrm{d} \mu+\frac{2}{\lambda} \int_{\partial_{r} M}\left(\partial_{r} \phi\right) \partial_{r}\left(\phi \psi^{-1}\right) \mathrm{d} \mu_{\partial}^{r} \\
& \geq \frac{2}{\lambda} \int_{M_{0, r}}\left(\phi \psi^{-1}\left[L, \partial_{r}\right] \phi-\phi\left(\partial_{r} \phi\right) L \psi^{-1}-2\left\langle\nabla \phi, \nabla \psi^{-1}\right\rangle \partial_{r} \phi\right) \mathrm{d} \mu \\
& \quad+\frac{2}{\lambda} \int_{\partial_{r} M} \psi\left(\partial_{r} \phi\right) \partial_{r}\left(\phi \psi^{-1}\right) \mathrm{d} \mu_{\partial} \\
& =: I_{1}+I_{2} .
\end{aligned}
$$


By (3.4) and (3.8), we obtain

$$
\left.\frac{2}{\lambda} \mid \int_{\partial M}\left\{\phi N^{2} \phi\right\}(\cdot, 0)\right) \mathrm{d} \mu_{\partial} \mid \leq \alpha_{1}
$$

for some constant $\alpha_{1}>0$. Next, since $\left[\partial_{r}, L\right]$ is a continuous second order differential operator on the compact domain $M_{0, \delta}, \mu\left(\phi^{2}\right)=1, \mu\left(|\nabla \phi|^{2}\right)=\lambda$ and (3.8) holds, we may find out constants $\alpha_{2}, \alpha_{3}>0$ such that

$$
\left|I_{1}\right| \leq \frac{\alpha_{2}}{\lambda} \int_{M}\left\{|\nabla \phi|^{2}+|\phi|\left(|\nabla \phi|+\left\|\operatorname{Hess}_{\phi}\right\|_{H S}\right)\right\} \mathrm{d} \mu \leq \alpha_{3} .
$$

Moreover, obviously

$$
I_{2}=\frac{2}{\lambda} \int_{\partial M}\left(\partial_{r} \phi\right)^{2} \mathrm{~d} \mu_{\partial}+\frac{2}{\lambda} \int_{\partial_{r} M}\left(\phi \partial_{r} \phi\right) \partial_{r} \psi^{-1} \mathrm{~d} \mu_{\partial}^{r} .
$$

Combining this with (3.11)-(3.13), we we arrive at $=$

$$
h^{\prime \prime}(r) \geq \frac{4}{\lambda} \int_{\partial M}\left(\partial_{r} \phi\right)^{2} \mathrm{~d} \mu_{\partial}-\alpha_{1}+\frac{2}{\lambda} \int_{\partial_{r} M}\left(\phi \partial_{r} \phi\right)\left(\partial_{r} \psi^{-1}\right) \mathrm{d} \mu_{\partial}^{r}, \quad r \in[0, \delta] .
$$

Since $h^{\prime}(r)=\frac{2}{\lambda} \int_{\partial M} \phi \partial_{r} \phi \mathrm{d} \mu_{\partial}$, by Cauchy-Schwarz inequality we obtain

$$
\frac{4}{\lambda} \int_{\partial M}\left(\partial_{r} \phi\right)^{2} \mathrm{~d} \mu_{\partial} \geq \frac{\left|h^{\prime}(r)\right|^{2}}{h(r)} .
$$

This together with (3.14) implies

$$
h^{\prime \prime}(r) \geq \frac{\left|h^{\prime}(r)\right|^{2}}{h(r)}-\alpha_{1}+\frac{2}{\lambda} \int_{\partial_{r} M}\left(\phi \partial_{r} \phi\right)\left(\partial_{r} \psi^{-1}\right) \mathrm{d} \mu_{\partial}^{r}, \quad r \in[0, \delta] .
$$

(2) Since $\left.N \phi^{2}\right|_{\partial M}=0, \psi^{-1} \in C_{b}^{\infty}\left(M_{0, \delta}\right)$, and

$$
\int_{\partial M}\left(\left|L \phi^{2}\right|+\left|\nabla \phi^{2}\right|\right) \leq 4 \lambda+2 \sqrt{\lambda}
$$

by the integration by parts formula, there exist constants $\alpha_{5}, \alpha_{6}>0$ such that

$$
\begin{aligned}
& \frac{2}{\lambda} \int_{\partial_{r} M}\left(\phi \partial_{r} \phi\right)\left(\partial_{r} \psi^{-1}\right) \mathrm{d} \mu_{\partial}^{r}=\frac{1}{\lambda} \int_{\partial_{r} M}\left\{\partial_{r}\left(\phi^{2} \partial_{r} \psi^{-1}\right)-\phi^{2} \partial_{r}^{2} \psi^{-1}\right\} \mathrm{d} \mu_{\partial}^{r} \\
& \geq-\frac{2}{\lambda} \int_{M_{0, r}}\left|L\left(\phi^{2} \partial_{r} \psi^{-1}\right)\right| \mathrm{d} \mu-\alpha_{5} h(r) \geq-\alpha_{6}-\alpha_{5} h(r) .
\end{aligned}
$$

This and (3.15) yield

$$
h^{\prime \prime}(r) \geq \frac{\left|h^{\prime}(r)\right|^{2}}{h(r)}-\alpha_{1}-\alpha_{6}-\alpha_{5} h(r), \quad r \in[0, \delta] .
$$


So, it suffices to find out a constant $c>0$ depending only on $L$ and $M_{0, \delta}$ such that

$$
h(r) \leq c, \quad r \in[0, \delta] .
$$

By (3.7) and $\mu\left(\phi^{2}\right)=1$, we have

$$
\int_{0}^{\delta} h(r) \mathrm{d} r=\frac{2}{\lambda} \int_{\partial M \times[0, \delta]} \phi^{2}(\theta, r)^{2} \mu_{\partial}(\mathrm{d} \theta) \mathrm{d} r \leq \frac{2 c_{0}}{\lambda} .
$$

So, there exists $r_{0} \in[0, \delta]$ such that

$$
h\left(r_{0}\right) \leq \frac{2 c_{0}}{\delta \lambda} \leq \frac{2 c_{0}}{\delta \lambda_{1}}:=c_{1},
$$

where $\lambda_{1}$ is the first non-trivial Neumann eigenvalue of $L$ on $M$. On the other hand, by Integration by parts formula and noting that $\partial_{r} \phi(\theta, r)=0$ for $r=0$, for $r \in[0, \delta]$ we have

$$
\begin{aligned}
\left|h^{\prime}(r)\right| & =\frac{1}{\lambda}\left|\int_{\partial_{r} M}\left(\partial_{r} \phi^{2}\right) \psi^{-1} \mathrm{~d} \mu_{\partial}^{r}\right|=\frac{1}{\lambda}\left|\int_{\partial M_{0, r}}\left(\partial_{r} \phi^{2}\right) \psi^{-1} \mathrm{~d} \mu_{\partial}^{0, r}\right| \\
& \leq \frac{1}{\lambda} \int_{M_{0, r}}\left(\left|\psi^{-1} L \phi^{2}\right|+\left|\left\langle\nabla \psi^{-1}, \nabla \phi^{2}\right\rangle\right|\right) \mathrm{d} \mu .
\end{aligned}
$$

Combining this with (3.16), we find out a constant $c_{2}>0$ such that

$$
\left|h^{\prime}(r)\right| \leq c_{2}, \quad r \in[0, \delta] .
$$

This together with (3.18) implies (3.17) for $c=c_{1}+\delta c_{2}$. Then the proof is finished.

Proof of Lemma 3.2. Due to Lemma 3.4, this result can be proved by modifying the argument in [1, Proof of Proposition 2.4]. Let $C$ be the constant in Lemma 3.4.

(1) We first prove that for large enough $\lambda>0$,

$$
r \in[0,2 \delta / 3] \text { with } h^{\prime}(r)>0 \text { implies }\left|h^{\prime}(r)\right|^{2}<4 C h(r) .
$$

If the assertion is not true, then there exists $r_{0} \in[0,2 \delta / 3]$ such that $h^{\prime}\left(r_{0}\right)>0$ and $\left|h^{\prime}\left(r_{0}\right)\right|^{2} \geq$ $4 C h\left(r_{0}\right)\left(1+h\left(r_{0}\right)\right)$. Then by Lemma 3.4

$$
\begin{aligned}
& \left.\frac{\mathrm{d}}{\mathrm{d} r}\left\{\left|h^{\prime}(r)\right|^{2}-4 C h(r)\right\}\right|_{r=r_{0}}=2 h^{\prime}\left(r_{0}\right) h^{\prime \prime}\left(r_{0}\right)-4 C h^{\prime}\left(r_{0}\right) \\
& \geq \frac{2\left(h^{\prime}\left(r_{0}\right)\right)^{3}}{h\left(r_{0}\right)}-6 C h^{\prime}\left(r_{0}\right)=2 C h^{\prime}\left(r_{0}\right) \geq 4 C \sqrt{C h\left(r_{0}\right)}>0 .
\end{aligned}
$$

So, there exists $\varepsilon \in\left(0, \delta-r_{0}\right)$ such that $h^{\prime}(r)>0$ and $\left|h^{\prime}(r)\right|^{2} \geq 4 C h(r)$ hold for $r \in\left[r_{0}, r_{0}+\varepsilon\right]$. By a continuity argument we conclude that

$$
h^{\prime}(r)>0,\left|h^{\prime}(r)\right|^{2} \geq 4 C h(r), \quad r \in\left[r_{0}, \delta\right] .
$$


Indeed, if not then

$$
r_{1}:=\inf \left\{r \in\left[r_{0}, \delta\right]:\left|h^{\prime}(r)\right|^{2}<4 C h(r)\right\} \in\left[r_{0}+\varepsilon, \delta\right] \subset\left(r_{0}, \delta\right] .
$$

We have $h^{\prime}(r)>0$ for $r \in\left[r_{0}, r_{1}\right]$ and $\left|h^{\prime}\left(r_{1}\right)\right|^{2}=4 C h\left(r_{1}\right)$, so that (3.20) holds for $r_{1}$ replacing $r_{0}$. Thus, due to continuity, there exists $r_{2} \in\left[r_{0}, r_{1}\right)$ such that

$$
\frac{\mathrm{d}}{\mathrm{d} r}\left\{\left|h^{\prime}(r)\right|^{2}-4 C h(r)\right\}>0, \quad r \in\left[r_{2}, r_{1}\right]
$$

Since by the definition of $r_{1}$ we have $\left|h^{\prime}\left(r_{2}\right)\right|^{2}-4 C h\left(r_{2}\right) \geq 0$, this implies

$$
\left|h^{\prime}\left(r_{1}\right)\right|^{2}-4 C h\left(r_{1}\right)=\sup _{r \in\left[r_{2}, r_{1}\right]}\left\{\left|h^{\prime}(r)\right|^{2}-4 C h(r)\right\}>0
$$

which contradicts to $\left|h^{\prime}\left(r_{1}\right)\right|^{2}=4 C h\left(r_{1}\right)$. So, (3.21) holds and thus,

$$
\frac{\mathrm{d}}{\mathrm{d} r} \sqrt{h(r)} \geq \sqrt{C}, \quad r \in\left[r_{0}, \delta\right]
$$

This implies $h(r) \geq C\left(r-r_{0}\right)^{2}$ for $r \in\left[r_{0}, \delta\right]$, and hence, by (3.7),

$$
\begin{aligned}
& \frac{C}{3}\left(\delta-\frac{2 \delta}{3}\right)^{3} \leq C \int_{r_{0}}^{\delta}\left(r-r_{0}\right)^{2} \mathrm{~d} r \\
& \leq \int_{0}^{\delta} h(r) \mathrm{d} r \leq \frac{c_{0}}{\lambda} \int_{M} \phi^{2} \mathrm{~d} \mu=\frac{c_{0}}{\lambda},
\end{aligned}
$$

which is impossible for large enough $\lambda$. The contradiction means that for large enough $\lambda>0$, (3.19) holds.

(2) We then prove that for large $\lambda>0$,

$$
\left|h^{\prime}(r)\right|^{2} \leq 5 C h(r), \quad r \in[0,2 \delta / 3] .
$$

By the Neumann condition we have $h^{\prime}(0)=0$, so that the inequality in (3.22) holds in a neighborhood of 0 . Thus, if (3.22) does not hold, then

$$
r_{2}:=\inf \left\{r \in[0,2 \delta / 3]:\left|h^{\prime}(r)\right|^{2}>5 C h(r)\right\} \in(0,2 \delta / 3]
$$

exists, and

$$
\left|h^{\prime}\left(r_{2}\right)\right|^{2}=5 C h\left(r_{2}\right),\left.\quad \frac{\mathrm{d}}{\mathrm{d} r}\left\{\left|h^{\prime}(r)\right|^{2}-5 C h(r)\right\}\right|_{r=r_{2}} \geq 0 .
$$

Combining this with (3.19), we obtain $h^{\prime}\left(r_{2}\right)<0$ and

$$
0 \leq\left.\frac{\mathrm{d}}{\mathrm{d} r}\left\{\left|h^{\prime}(r)\right|^{2}-5 C h(r)\right\}\right|_{r=r_{2}}=2 h^{\prime}\left(r_{2}\right) h^{\prime \prime}\left(r_{2}\right)-5 C h^{\prime}\left(r_{2}\right)=2 h^{\prime}\left(r_{2}\right)\left(h^{\prime \prime}\left(r_{2}\right)-\frac{5 C}{2}\right) .
$$

So, $h^{\prime \prime}\left(r_{2}\right) \leq \frac{5 C}{2}$. But by Lemma 3.4 and $\left|h^{\prime}\left(r_{2}\right)\right|^{2}=5 C h\left(r_{2}\right)$ we have

$$
h^{\prime \prime}\left(r_{2}\right) \geq \frac{\left|h^{\prime}\left(r_{2}\right)\right|^{2}}{h\left(r_{2}\right)}-C=4 C,
$$


which is a contradiction. Therefore, (3.22) has to be true.

(3) By (3.22), when $\lambda>0$ is large enough we have

$$
\frac{\mathrm{d}}{\mathrm{d} r} \sqrt{h(r)}=\frac{h^{\prime}(r)}{2 \sqrt{h(r)}} \geq-\frac{\sqrt{5 C h(r)}}{2 \sqrt{h(r)}}=-\frac{\sqrt{5 C}}{2}=:-c, \quad r \in[0,2 \delta / 3] .
$$

So, $\sqrt{h(r)} \geq \sqrt{h(0)}-\sqrt{c} r$ holds for $r \in[0,2 \delta / 3]$. Let $M=\frac{16\left(1 \vee c_{0}\right)}{c}$, where $c_{0}$ is in (3.7). If $h(0) \geq M c \lambda^{-2 / 3}$, we would have

$$
\sqrt{h(r)} \geq \sqrt{M c}\left(\lambda^{-1 / 3}-r\right) \geq \frac{\lambda^{-1 / 3}}{2} \sqrt{M c}, \quad r \in\left[0, \lambda^{-1 / 3} / 2\right]
$$

where we take $\lambda>0$ large enough such that $\lambda^{-1 / 3} / 2 \leq 2 \delta / 3$. Combining this with (3.7) and (3.9), we arrive at

$$
\begin{aligned}
\frac{2 c_{0}}{\lambda} & \leq \frac{M c}{8 \lambda} \leq \int_{0}^{\lambda^{-1 / 3} / 2} h(r) \mathrm{d} r \leq \frac{1}{\lambda} \int_{\partial M \times[0, \delta]} \phi(\theta, r)^{2} \mu_{\partial}(\mathrm{d} \theta) \mathrm{d} r \\
& \leq \frac{c_{0}}{\lambda} \int_{M} \phi^{2} \mathrm{~d} \mu=\frac{c_{0}}{\lambda}
\end{aligned}
$$

for large enough $\lambda>0$, which is however impossible. This means that when $\lambda>0$ is large enough we have $h(0) \leq M c \lambda^{-2 / 3}$, equivalently,

$$
\int_{\partial M} \phi^{2} \mathrm{~d} \mu_{\partial} \leq M c \lambda^{1 / 3}
$$

which completes the proof.

We are now ready to prove Theorem 1.2.

Proof of Theorem 1.2. Since $L \phi=-\lambda \phi$, estimate (1.6) follows from (2.1). Moreover, estimates in (1.7) are included in Lemma 3.1 and Lemma 3.2. Combining (1.6) with (1.7) we prove (1.8).

\section{References}

[1] A. Barnett, A. Hassell, M. Tacy, Comparable upper and lower bounds for boundary values of Neumann eigenfunctions and tight inclusion of eigenvalues, arXiv:1512.04165v2.

[2] A. Hassell, T. Tao, Upper and lower bounds for normal derivatives of Dirichlet eigenfunctions, Math. Res. Lett. 9(2002); Erratum 17(2010).

[3] F.-Y. Wang, Second fundamental form and gradient of Neumann semigroups, J. Funct. Anal. 256(2009), 3461-3469.

[4] F.-Y. Wang, Identifying constant curvature manifolds, Einstein manifolds, and Ricci parallel manifolds, arXiv:1710.00276. 